\title{
Darmkrebsprävention
}

\section{Ist der herkömmliche Stuhltest überholt?}

Immunologische Stuhltests statt des traditionellen Guajak-Tests fordert die Stiftung Lebensblicke in ihrer Hamburger Erklärung. Goldstandard der Kolonund Rektumkarzinomprävention soll aber die Darmspiegelung bleiben. Wir fragten nach bei Dr. Gerhard Brenner, Vorstandsmitglied der Stiftung.

MMW: Wie ist die gesetzliche Ausgangs lage, welche Leistungen zur Darmkrebsprävention übernehmen die Kassen, welche nicht?

Brenner: Im Jahr 2002 wurde das gesetzliche Krebsfrüherkennungsprogramm durch die Einführung der präventiven Darmspiegelung ergänzt. Gesetzlich Krankenversicherte können ab 55 Jahren diese Vorsorgekoloskopie bei einem Gastroenterologen kostenlos durchführen lassen. Liegt kein pathologischer Befund vor, sollte die Vorsorgekoloskopie im Abstand von zehn Jahren wiederholt werden. Mit der Koloskopie kann nicht nur ein bestehender Darmkrebs früh erkannt werden, sondern insbesondere mögliche Vorstufen wie Darmpolypen oder Adenome. Diese werden in einem Arbeitsgang bei der diagnostischen Maßnahme abgetragen.

Personen im Alter von 50-54 Jahren können - wie bisher - jährlich den Test auf verstecktes Blut im Stuhl machen. Versicherten im Alter ab 55 Jahren, die sich nicht für die Vorsorgekoloskopie entschei-

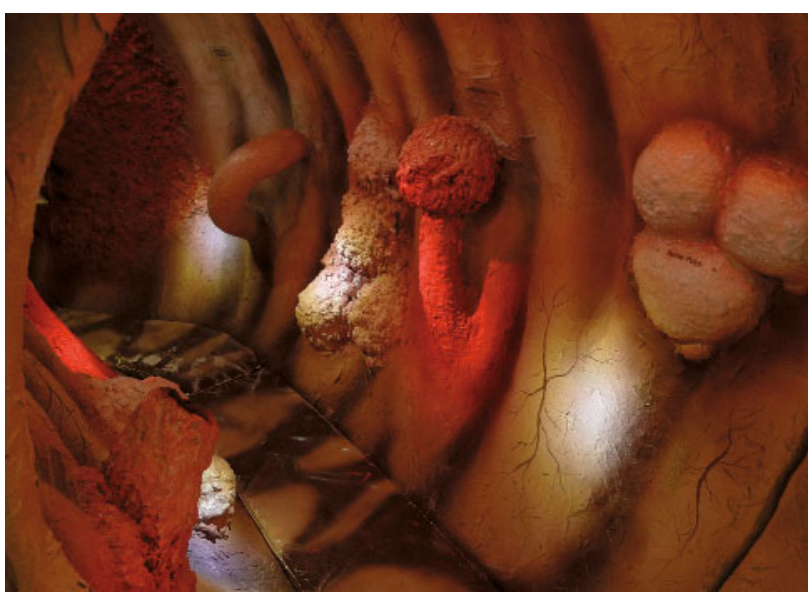

Im begehbaren Darm, ausgestellt auf der Tagung der Viszeralmediziner, ist die Vielfalt der Darmpolypen plastisch dargestellt. den, wird der Stuhltest im zweijährigen Abstand angeboten.

Ein positiver Stuhltestbefund sagt lediglich etwas über eine Blutungsquelle aus, deren Ursachen dann durch eine Darmspiegelung abzuklären sind. Die Aussagefähigkeit der Stuhltests, insbesondere der chemischen guajakbasierten Stuhltests, die seit 1971 im gesetzlichen Früherkennungsprogramm eingesetzt werden, ist beschränkt. Die Vorsorgekoloskopie wird jährlich von etwa 550000 Versicherten über 55 Jahren in Anspruch genommen. Aber rund 4,5 Millionen Personen machen immer noch den Stuhltest.

MMW: Der Guajak-Test wird empfohlen, weil evidenzbasierte Belege für die Wirksamkeit vorliegen. Gibt es auf diesem Gebiet keine Fortschritte, die evidenzbasiert begründet werden können? Brenner: Die Stiftung Lebensblicke fordert die Zulassung der immunologischen Stuhltests im gesetzlichen Krebsfrüherkennungsprogramm, weil sie eine weit bessere Sensitivität und Spezifität als der Guajak-Test haben. Diese Tests sind seit mehreren Jahren von verschiedenen Herstellern auf dem Markt. Sie können gegenwärtig aber von gesetzlich Versicherten nur als Wahlleistung auf Selbstzahlerbasis nachgefragt werden. Zahlreiche nationale und internationale Studien haben die Vorteilhaftigkeit dieser immunologischen bei Dr. med. Gerhard Brenner

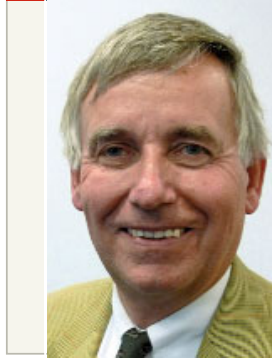

„Die Aussagefähigkeit der Stuhltests, besonders des fast 40 Jahre alten guajakbasierten Stuhltests, ist beschränkt."
Stuhltests hinsichtlich ihrer treffsicheren Aussagefähigkeit gegenüber dem fast 40 Jahre alten Guajaktest belegt.

MMW: Die Stiftung hat eine Studie zur Darmprävention aufgelegt. Wie sind die Ergebnisse ausgefallen?

Brenner: Die Stiftung Lebensblicke hat die Schirmherrschaft für verschiedene Studien zum Ergebnisvergleich von guajakbasierten und immunologischen Tests übernommen. Die Ergebnisse wurden anlässlich des Kongresses der Deutschen Gesellschaft für Stoffwechsel- und Verdauungsstörungen vorgestellt. Die Meinung der wissenschaftlichen Studienleiter fiel einheitlich zugunsten der immunologischen Stuhltests aus. Die Kostenerstattung sollte den gesetzlich Versicherten nicht länger vorenthalten werden. Das ist im Wesentlichen der Inhalt der Hamburger Erklärung der Stiftung Lebensblicke. Dabei ist allerdings zu bemerken, dass die Darmspiegelung der Goldstandard für die Darmkrebsprävention bleibt. Auch der immunologische Test kann nur als zweitbeste Maßnahme angesehen werden für die Personen, die den Stuhltest - aus welchen Gründen auch immer-vorziehen.

MMW: Wann sollte eine Koloskopie zur Prävention durchgeführt werden? Muss immer ein Bluttest im Stuhl vorausgehen? Brenner: Im Alter $a b 55$ Jahren und bei befundfreiem Ergebnis ist eine Wiederholung nach zehn Jahren zu empfehlen. Sind Polypen oder Adenome gefunden und abgetragen worden, ist eine Kontrollspiegelung in kürzeren Abständen erforderlich. Ein Stuhltest vor der Vorsorgekoloskopie ist in Deutschland nicht erforderlich.

- Interview: Dr. med. Jochen Aumiller 